\title{
Survival after out-of-hospital cardiac arrest is associated with area-level socioeconomic status
}

\author{
Martin Jonsson, ${ }^{1}$ Juho Härkönen, ${ }^{2}$ Petter Ljungman, ${ }^{3}$ Araz Rawshani, ${ }^{4}$ Per Nordberg, ${ }^{1}$ \\ Leif Svensson, ${ }^{1}$ Johan Herlitz, ${ }^{5}$ Jacob Hollenberg ${ }^{1}$
}

- Additional material is published online only. To view please visit the journal online (http://dx.doi.org/10.1136/ heartjnl-2018-313838)

${ }^{1}$ Center for Resuscitation Science, Department for Medicine, Karolinska Institutet, Stockholm, Sweden ${ }^{2}$ Department of Political and Social Sciences, European University Institute, Florence, Italy

${ }^{3}$ Institute of Environmental Medicine, Karolinska Institutet, Stockholm, Sweden

${ }^{4}$ Department of Molecular and Clinical Medicine, Gothenburg University, Gothenburg, Sweden ${ }^{5}$ Center for Pre-Hospital Research, University of Borås, Borås, Sweden

\section{Correspondence to}

Martin Jonsson, Centre for Resuscitation Science, Department of Medicine, Karolinska Institutet, Stockholm 118 83, Sweden; martin.k. jonsson@ki.se

Received 6 July 2018 Revised 18 September 2018 Accepted 22 September 2018
Objective Out-of-hospital cardiac arrest (OHCA) is a major cause of death in the Western world. In this study we aimed to investigate the relationship between arealevel socioeconomic status (SES) and 30-day survival after OHCA. We hypothesised that high SES at an area level is associated with an improved chance of 30-day survival.

Methods Patients with OHCA in Stockholm County between 1 January 2006 and 31 December 2015 were analysed retrospectively. To quantify area-level SES, we linked the patient's home address to $250 \times 250 / 1000$ $\times 1000$ meter grids with aggregated information about income and education. We constructed multivariable logistic regression models in which area-level SES measures were adjusted for age, sex, emergency medical services response time, witnessed status, initial rhythm, aetiology, location and year of cardiac arrest.

Results We included 7431 OHCAs. There was significantly greater 30 -day survival $(p=0.003)$ in areas with a high proportion of university-educated people. No statistically significant association was seen between median disposable income and 30-day survival. The adjusted OR for 30-day survival among patients in the highest educational quintile was $1.70(95 \% \mathrm{Cl} 1.15$ to 2.51) compared with patients in the lowest educational quintile. We found no significant interaction for sex. Positive trend with increasing area-level education was seen in both men and women but the trend was only statistically significant among men $(p=0.012)$ Conclusions Survival to 30 days after OHCA is positively associated with the average educational level of the residential area. Area-level income does not independently predict 30-day survival after OHCA.

\section{INTRODUCTION}

Out-of-hospital cardiac arrest (OHCA) is one of the leading causes of death in the Western world. Of the approximately 300000 emergency medical services (EMS) cases of OHCA treated annually in Europe, only about $10 \%$ survive to 30 days. ${ }^{1}$ Survival is related to several resuscitation-associated factors such as shockable initial rhythm, bystander cardiopulmonary resuscitation (CPR) and witnessed arrest. ${ }^{2}$

Socioeconomic status (SES) is a multidimensional concept that reflects one's position in a social hierarchy. Income and education are two commonly used measures of SES which are associated with both the incidence/prevalence of and survival from cardiovascular disease. ${ }^{34}$ Income and education are often used interchangeably, although they can tap into different proximate determinants of health. ${ }^{56}$ The relationship between SES and cardiovascular outcomes has also been reported to vary according to sex. ${ }^{7}$ Compared with cardiovascular disease in general, only a few studies have focused on the association between SES and survival after OHCA. The results have been contradictory, and while most previous studies have revealed a positive relationship between SES and survival, ${ }^{8-12}$ others have not. $^{1314}$

As survival rates in cases of OHCA are low, ${ }^{1}$ knowledge of factors that can predict survival are of foremost importance. SES variables are readily available and can therefore be used in connection with targeted interventions aimed at vulnerable groups, in order to reduce disparities.

The aim of this study was to assess the chance of survival after OHCA in relation to SES. We hypothesised that residing in a neighbourhood with a relatively high average level of education or average income would predict better 30-day survival after OHCA. We also investigated whether there was any relation to gender.

\section{METHODS}

\section{Study setting}

Stockholm County covers $6517 \mathrm{~km}^{2}$ and had 2163042 inhabitants in $2013 .{ }^{15}$ The EMS system is two-tiered in high-priority emergencies such as OHCAs. The first tier can provide basic life support, while the second tier can provide advanced life support (by a nurse anaesthetist or an anaesthesiologist). In addition, the fire brigade and police are dispatched in cases of suspected OHCA.

\section{The Swedish Registry for Cardiopulmonary Resuscitation}

Cardiac arrest data were retrieved from the Swedish Registry for Cardiopulmonary Resuscitation (SRCR). SRCR holds a nationwide quality register with support from the Swedish Association of Local Authorities and Regions. All EMS services in Sweden report to the registry. EMS personnel collected data prospectively after OHCAs in a predefined manner according to the Utstein template during the whole period. ${ }^{16}$ Follow-up was conducted by linking SRCR to the Swedish National Population Registry.

\section{Selection of patients}

We included all EMS-treated cases of OHCA between 1 January 2006 and 31 December 2015. 


\section{OHCA in Stockholm 2006-2015 \\ $\mathrm{n}=9791$}

\section{Missing ID / living outside Stockholm $\mathrm{n}=1011$}

\section{Residents in Stockholm $n=8780$}

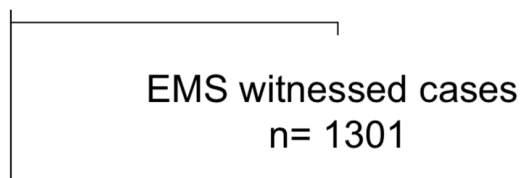

Non-EMS witnessed
\[ n=7479 \]

Missing SES information $n=48$

\section{Analytic sample $n=7431$}

Figure 1 Flow chart of patient selection. OHCA, out-of-hospital cardiac arrest; EMS, emergency medical services; SES, socioeconomic status.

Patients with unknown identity and patients residing outside Stockholm County were not eligible for inclusion (not possible to link them to the grid). EMS-witnessed cases were excluded as a result of missing information on bystander CPR and invalid response times.

\section{Area-level SES}

Area-level SES was quantified by using grids of $250 \times 250$ metres in urban areas, and $1000 \times 1000$ metres in rural areas (figure 1). These grid areas are predefined by Statistics Sweden and were downloaded from https://zeus.slu.se/ a service offered to Swedish universities. The home addresses of the OHCA patients were geo coded and data were merged with the grid layer, whereby area-level income and education could be assigned to the individual (R package $\mathrm{sp}$ ).

The area-level SES variables used in the analyses were median disposable family income and the percentage of the population with a university degree (bachelor's or above) in 2013, categorised into quintiles within the grids.

\section{Statistical analysis}

All statistical analyses were conducted by using R V.3.4.3 for Mac OSx (R Foundation for Statistical Computing, 2017). In the descriptive analysis, baseline variables are presented as counts and proportions. We used the $\chi^{2}$ trend test for differences in proportions, and the Jonckheere-Terpstra test for differences in medians between groups.
Multivariable logistic regression analyses were used to analyse the relationships between area-level education and income, and 30-day survival after OHCA. Adjustment was made for important covariates, namely sex, age (in years), witnessed status (witnessed or not witnessed), initial rhythm (shockable vs non-shockable), aetiology (cardiac vs non-cardiac), response time (call to arrival at scene), location (outside home vs at home), year of cardiac arrest, bystander CPR, (yes/no) and population density (in quartiles). Three models were specified; the first included only education as an SES measure, the second included only income as an SES measure, and the third included both education and income as SES measures.

To account for incomplete cases $(\mathrm{n}=532,7.2 \%)$, missing data were imputed using multiple imputations by chained equations. ${ }^{17}$ Missing data were assumed to be missing at random and 15 data sets were generated using the mice package in $\mathrm{R}$. The multivariable logistic regression analysis specified above was conducted in all 15 data sets and the results were pooled using Rubin's rules.

Variance-inflated factors (VIFs) were calculated to test for multicollinearity ( $\mathrm{R}$ package $\mathrm{rms}$ ). The Hosmer-Lemeshow goodness-of-fit test was used to test model fit ( $\mathrm{R}$ package ResourceSelection).

\section{RESULTS}

Between 1 January 2006 and 31 December 2015 a total of 9791 OHCAs occurred in Stockholm County. Patients living outside Stockholm County (eg, in another city or abroad) or of unknown identity were not eligible for the study $(n=1011)$. One thousand three hundred and one cases were witnessed by EMS and were excluded as a result of difficulties in using these cases in the regression models (no information on bystander CPR). Hence, 7479 cases remained eligible for inclusion. We also then excluded cases with incomplete information on the area-level education and income $(\mathrm{n}=48)$. The final analytical sample included 7431 cases (figure 1).

\section{Baseline characteristics of patients grouped by area-level education}

Differences in baseline characteristics between areas grouped by area-level education are presented in table 1 . The greatest differences between the areas were found in age (67 years in the lowest quintile vs 74 years in the highest quintile of average education), proportion of witnessed arrests $(58.4 \%$ vs $62.2 \%)$, EMS response time (12 $\mathrm{min}$ vs $9 \mathrm{~min}$ ) and population density (for further details see online table $\mathrm{S} 1$ in supplementary appendix).

\section{Baseline characteristics of patients grouped by area-level income}

Categorised by income, we found significant differences between the areas in all baseline variables except the location of arrest (table 2). Between areas with the lowest and highest median incomes, respectively, the largest differences were noted for female sex (37.2\% vs $29.8 \%)$, witnessed arrest $(57.2 \%$ vs $63.5 \%)$, ventricular fibrillation/ventricular tachycardia) $(19.4 \%$ vs $24.7 \%)$ and CPR prior to EMS arrival $(59.4 \%$ vs $65.8 \%)$ and population density.

\section{Crude survival rates}

Crude survival rates among patients grouped by area-level education are presented in figure 2, panel A. We found significant trends in survival after 1 day $(20.4 \%$ vs $23.0 \%$, p for trend $=0.024)$, at 30 days $(6.5 \%$ vs $9.6 \%$, p for trend $<0.001)$ and after 1 year $(6.1 \%$ vs $8.7 \%$, p for trend 0.001$)$. 
Table 1 Baseline variables of patients according to area-level education

\begin{tabular}{|c|c|c|c|c|c|c|}
\hline Education & Q1 & Q2 & Q3 & Q4 & Q5 & $P$ for trend \\
\hline $\mathrm{n}$ & 1485 & 1487 & 1486 & 1488 & 1485 & \\
\hline Age (median, (Q1, Q3)) & $67(54,78)$ & $70(58,80)$ & $72(61,82)$ & $71(60,82)$ & $74(62,84)$ & $<0.001$ \\
\hline Female sex $(n, \%)$ & $514(34.6)$ & $540(36.3)$ & $521(35.1)$ & $482(32.4)$ & $513(34.5)$ & 0.298 \\
\hline Witnessed $(n, \%)$ & $861(58.4)$ & $862(58.4)$ & $877(59.3)$ & $877(59.2)$ & $918(62.2)$ & 0.032 \\
\hline VF/VT $(n, \%)$ & $324(21.9)$ & $313(21.1)$ & $333(22.5)$ & $326(22.0)$ & $341(23.1)$ & 0.354 \\
\hline Cardiac aetiology $(\mathrm{n}, \%)$ & $1163(78.4)$ & $1176(79.1)$ & $1187(79.9)$ & $1187(79.8)$ & $1184(79.7)$ & 0.290 \\
\hline Response time (median, (Q1,Q3)) & $12(8,17)$ & $11(8,15)$ & $11(8,14)$ & $10(7,14)$ & $9(7,13)$ & $<0.001$ \\
\hline At home $(n, \%)$ & $1061(71.4)$ & $1046(70.4)$ & $1083(72.9)$ & $1065(71.6)$ & $1048(70.6)$ & 0.889 \\
\hline CPR prior to EMS $(n, \%)$ & $948(63.8)$ & $908(61.1)$ & $929(62.5)$ & $933(62.7)$ & $942(63.4)$ & 0.834 \\
\hline \multicolumn{7}{|l|}{ Population density (quartiles) } \\
\hline Q1 (n, \%) & $370(28.1)$ & $271(18.8)$ & $375(26.0)$ & $375(25.7)$ & $369(25.1)$ & 0.005 \\
\hline Q2 (n, \%) & $330(25.0)$ & $414(28.7)$ & $444(30.7)$ & $373(25.5)$ & $248(16.9)$ & $<0.001$ \\
\hline Q3 (n, \%) & $354(26.8)$ & $495(34.3)$ & $426(29.5)$ & $345(23.6)$ & $164(11.2)$ & $<0.001$ \\
\hline Q4 (n, \%) & $265(20.1)$ & $264(18.3)$ & $199(13.8)$ & $368(25.2)$ & $689(46.9)$ & $<0.001$ \\
\hline
\end{tabular}

CPR, cardiopulmonary resuscitation; EMS, emergency medical services; VF/VT, ventricular fibrillation/ventricular tachycardia; Q, quintile.

A similar pattern was seen among patients grouped by arealevel income (figure 2, panel B). The survival difference between the lowest and highest income groups was $21.5 \%$ vs $24.4 \%$ after 1 day ( $\mathrm{p}$ for trend $=0.021$ ), $6.3 \%$ vs $10.5 \%$ after 30 days ( $\mathrm{p}$ for trend $<0.001$ ) and 5.8 vs 9.5 after 1 year ( $p$ for trend $<0.001$ ).

\section{Adjusted survival rates}

Results from the regression analysis are presented in table 3 . In model 1, where area-level education and patient/resuscitation covariates were included, we saw a significant positive trend between area-level education and the probability of 30-day survival ( $\mathrm{p}$ for trend $<0.001$ ). The highest estimate was found in areas with the highest educational levels (Q5), with an adjusted OR for 30-day survival of 1.93 (95\% CI 1.41 to 2.64) compared with the lowest quintile of education.

In model 2, area-level income and patient/resuscitation covariates were included. There was a positive trend as regards arealevel income ( $\mathrm{p}$ for trend $<0.001$ ). The adjusted OR for 30 -day survival among individuals in the highest income quintile was 1.88 (95\% CI 1.36 to 2.59 ), compared with those in the lowest income quintile.

When both area-level education and income were included in the mutually adjusted model (model 3 ), there was a significantly positive trend for 30-day survival with increased educational level ( $\mathrm{p}$ for trend $=0.003$ ). There was a stepwise increase in 30-day survival for each area-level education quintile. The residents of the highest educated areas had the greatest odds of survival, with an OR of 1.70 (95\% CI 1.15 to 2.51). Regarding income, there was no statistically significant relationship between area-level income and 30-day survival ( $\mathrm{p}$ for trend=0.388). Residents of the highest average income areas had the highest survival odds, with an OR for 30-day survival of 1.31 (95\% CI 0.87 to 1.98).

\section{Sex-stratified analyses}

Interaction between sex and the SES variables was not significant. The interaction term for sex and education had a $\mathrm{p}$ value of 0.825 , while that for sex and income had a $p$ value of 0.323 .

Figure $3 \mathrm{~A}$ shows the estimates for area-level education separately for men and women. For men $(n=4861)$, there was a significantly positive trend $(\mathrm{p}=0.012)$ between area-level education and survival, the OR for 30-day survival among men living in areas with the highest level of education was $1.69,(95 \% \mathrm{CI}$ 1.06 to 2.70$)$. For women $(n=2570)$, the trend between arealevel education and 30-day survival followed a positive gradient but was not statistically significant $(\mathrm{p}=0.227)$.

Table 2 Baseline variables of patients according to area-level income

\begin{tabular}{|c|c|c|c|c|c|c|}
\hline Income & Q1 & Q2 & Q3 & Q4 & Q5 & $\mathrm{P}$ for trend \\
\hline $\mathrm{n}$ & 1486 & 1485 & 1488 & 1487 & 1485 & \\
\hline Age (median, (Q1, Q3)) & $67(55,79)$ & $72(60,82)$ & $73(61,83)$ & $72(61,82)$ & $70(60,81)$ & $<0.001$ \\
\hline Female sex $(n, \%)$ & $553(37.2)$ & $571(38.5)$ & $533(35.8)$ & $470(31.6)$ & $443(29.8)$ & $<0.001$ \\
\hline Witnessed (n, \%) & $842(57.2)$ & $850(57.6)$ & $877(59.3)$ & $890(60.0)$ & $936(63.5)$ & $<0.001$ \\
\hline VF/VT (n, \%) & $287(19.4)$ & $287(19.4)$ & $328(22.1)$ & $370(24.9)$ & $365(24.7)$ & $<0.001$ \\
\hline Cardiac aetiology $(\mathrm{n}, \%)$ & $1140(76.8)$ & $1186(79.9)$ & $1179(79.2)$ & $1203(80.9)$ & $1189(80.1)$ & 0.020 \\
\hline Response time (median, (Q1,Q3)) & $10(8,15)$ & $11(8,16)$ & $10(7,15)$ & $10(7,15)$ & $10(7,14)$ & 0.016 \\
\hline At home $(n, \%)$ & $1072(72.1)$ & 1062 (71.5) & $1026(69.0)$ & 1068 (71.8) & $1075(72.4)$ & 0.828 \\
\hline CPR prior to EMS (n, \%) & $882(59.4)$ & $899(60.5)$ & $924(62.1)$ & $978(65.8)$ & $977(65.8)$ & $<0.001$ \\
\hline \multicolumn{7}{|l|}{ Population density (quartiles) } \\
\hline Q1 (n, \%) & $182(12.5)$ & $240(16.7)$ & $262(18.4)$ & $420(30.1)$ & $656(46.3)$ & $<0.001$ \\
\hline $\mathrm{Q} 2(\mathrm{n}, \%)$ & $270(18.5)$ & $420(29.2)$ & $421(29.5)$ & $369(26.4)$ & $329(23.2)$ & 0.200 \\
\hline Q3 (n, \%) & $496(34.0)$ & $563(39.2)$ & $452(31.7)$ & $185(13.2)$ & $88(6.2)$ & $<0.001$ \\
\hline $\mathrm{Q} 4(\mathrm{n}, \%)$ & $510(35.0)$ & $215(15.0)$ & $292(20.5)$ & $423(30.3)$ & $345(24.3)$ & 0.019 \\
\hline
\end{tabular}

CPR, cardiopulmonary resuscitation; EMS, emergency medical services; OHCA, out of-hospital cardiac arrest; VF/VT, ventricular fibrillation/ventricular tachycardia; Q, quintile. 
A. Areas categorised by education

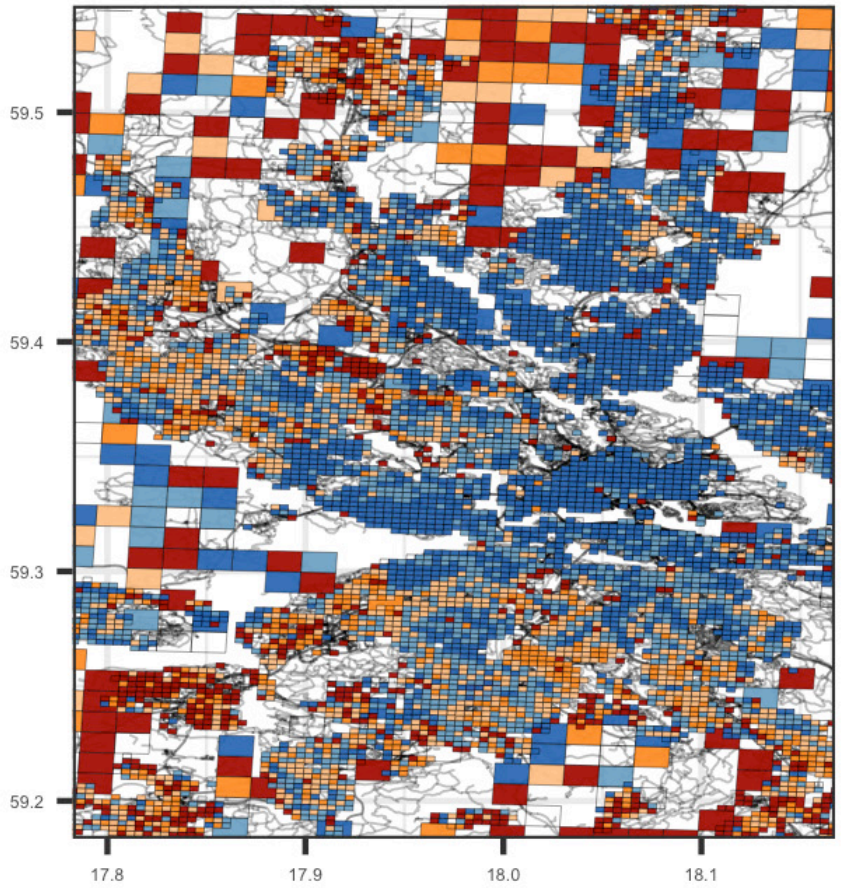

B. Areas categorised by income

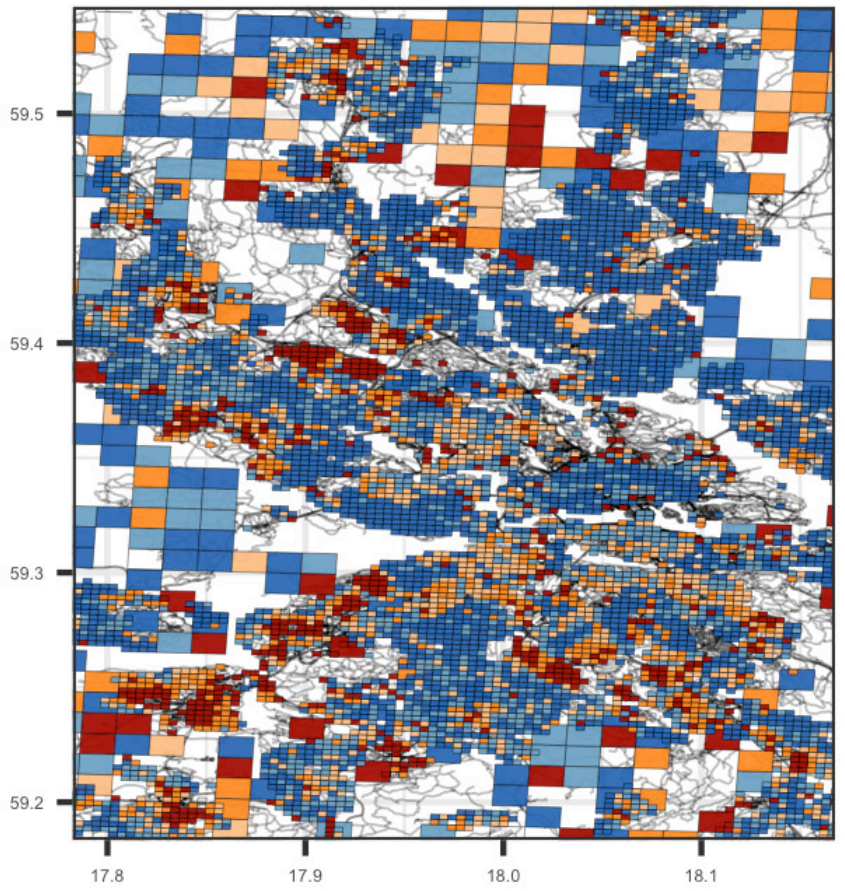

Day 1

Day 30

Day 365

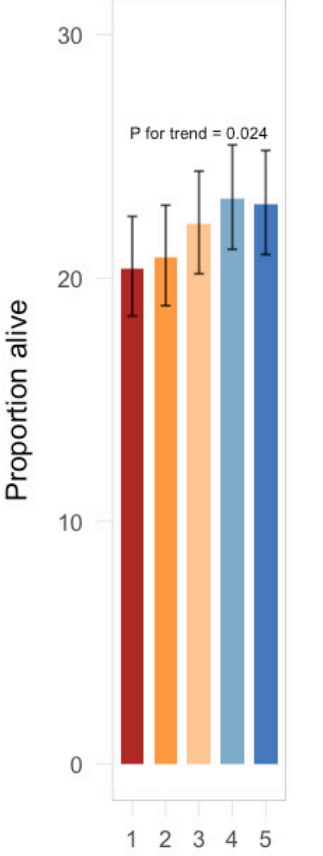

30

30

20

20

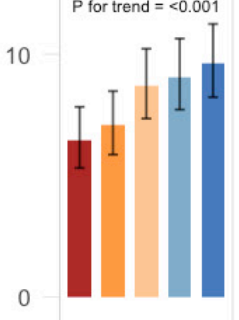

10

P for trend $=0.001$

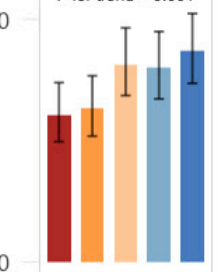

$\begin{array}{lllll}1 & 2 & 3 & 4 & 5\end{array}$

Education quintiles

Q1 (low) Q2 Q Q3 Q4 Q

Day 1

Day 30

Day 365

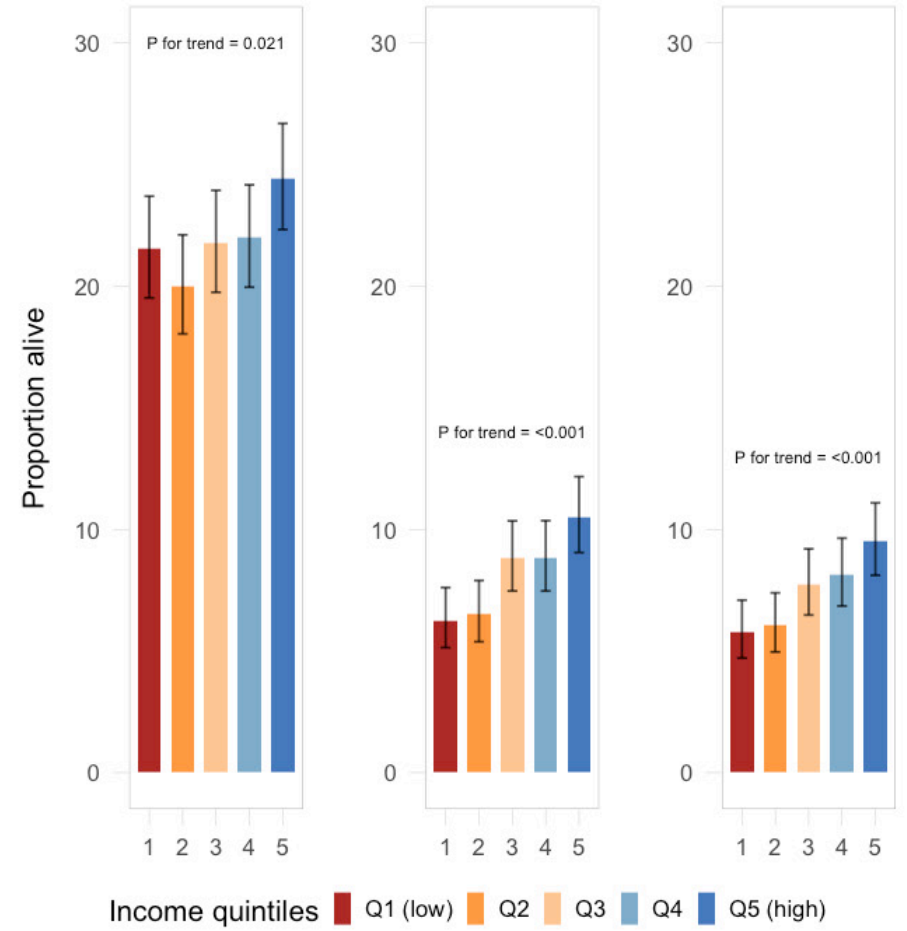

Figure 2 Maps of the $250 \times 250 / 1000 \times 1000$ meter grid and crude survival rates. Panel A, categorised by education quintiles; Panel B, categorised by income quintiles.

Figure $3 \mathrm{~B}$ presents the relationship between area-level income quintiles and 30-day survival for men and women. Most of the estimates were not statistically significant and there was no statistically significant trend for either sex.

\section{Model fit}

The correlation between area-level income and area-level education was 0.63 . There were no signs of problematic multicollinearity in any of the models. The highest VIF value was found in connection with area-level income (quintile 5) in the analysis 
Table 3 Logistic regression for 30-day survival after multiple imputations

\begin{tabular}{|c|c|c|c|}
\hline & Model 1 & Model 2 & Model 3 \\
\hline & OR $(95 \% \mathrm{Cl})$ & OR $(95 \% \mathrm{Cl})$ & OR $(95 \% \mathrm{CI})$ \\
\hline Education Q1 & $1.0(<0.001)^{*}$ & & $1.0(0.003)^{*}$ \\
\hline Education Q2 & 1.27 (0.92 to 1.75$)$ & & 1.24 (0.90 to 1.72$)$ \\
\hline Education Q3 & 1.65 (1.21 to 2.24$)$ & & 1.53 (1.10 to 2.14$)$ \\
\hline Education Q4 & 1.76 (1.30 to 2.40$)$ & & 1.61 (1.13 to 2.28$)$ \\
\hline Education Q5 & 1.93 (1.41 to 2.64$)$ & & 1.70 (1.15 to 2.51$)$ \\
\hline Income Q1 & & $1.0(<0.001)^{*}$ & $1.0(0.388)^{*}$ \\
\hline Income Q2 & & 1.34 (0.96 to 1.87$)$ & 1.18 (0.83 to 1.66$)$ \\
\hline Income Q3 & & 1.56 (1.14 to 2.14$)$ & 1.23 (0.87 to 1.75$)$ \\
\hline Income Q4 & & 1.49 (1.08 to 2.05$)$ & 1.12 (0.77 to 1.63$)$ \\
\hline Income Q5 & & 1.88 (1.36 to 2.59$)$ & 1.31 (0.87 to 1.98$)$ \\
\hline Female sex & $1.23(0.99$ to 1.53$)$ & $1.23(0.99$ to 1.53$)$ & 1.23 (0.99 to 1.54$)$ \\
\hline Age & 0.97 (0.96 to 0.98$)$ & 0.97 (0.97 to 0.98$)$ & 0.97 (0.96 to 0.97$)$ \\
\hline At home & 0.45 (0.37 to 0.54$)$ & 0.45 (0.37 to 0.54$)$ & $0.45(0.37$ to 0.54$)$ \\
\hline Response time (per min) & 0.97 (0.96 to 0.99$)$ & 0.97 (0.96 to 0.99$)$ & 0.97 (0.96 to 0.99$)$ \\
\hline VF/VT & 9.29 (7.42 to 11.62$)$ & 9.2 (7.36 to 11.51$)$ & 9.3 (7.43 to 11.64$)$ \\
\hline Witnessed OHCA & 2.83 (2.20 to 3.65$)$ & 2.8 (2.17 to 3.62$)$ & 2.83 (2.19 to 3.65 ) \\
\hline Non-cardiac aetiology & $1.31(0.96$ to 1.80$)$ & 1.31 (0.95 to 1.79$)$ & 1.31 (0.96 to 1.8$)$ \\
\hline Bystander CPR & 1.39 (1.10 to 1.76$)$ & 1.37 (1.09 to 1.74$)$ & 1.39 (1.10 to 1.76$)$ \\
\hline Year of OHCA & 1.10 (1.07 to 1.14$)$ & 1.1 (1.06 to 1.14$)$ & 1.1 (1.07 to 1.14$)$ \\
\hline Population density (Q1) & 1.0 & 1.0 & 1.0 \\
\hline Population density (Q2) & $0.97(0.75$ to 1.26$)$ & 1.04 (0.79 to 1.35$)$ & 0.99 (0.76 to 1.3$)$ \\
\hline Population density (Q3) & 1.04 (0.79 to 1.36$)$ & 1.18 (0.88 to 1.58$)$ & 1.09 (0.81 to 1.46$)$ \\
\hline Population density (Q4) & $0.84(0.64$ to 1.10$)$ & 1.02 (0.77 to 1.34$)$ & $0.9(0.67$ to 1.21$)$ \\
\hline
\end{tabular}

* $p$ for trend.

CPR, cardiopulmonary resuscitation; OHCA, out of-hospital cardiac arrest; VF/VT, ventricular fibrillation/ventricular tachycardia; Q, quintile.

that was restricted to men $(\mathrm{VIF}=3.72)$. (For all VIFs, see online table S2 in supplementary appendix) The Hosmer-Lemeshow goodness-of-fit test was not significant in any of the models.

\section{Spatial autocorrelation}

Although not the primary analysis, we tested the areas for spatial autocorrelation using Moran's I test. The Moran's I statistic was $0.0166(\mathrm{p}=0.371)$.

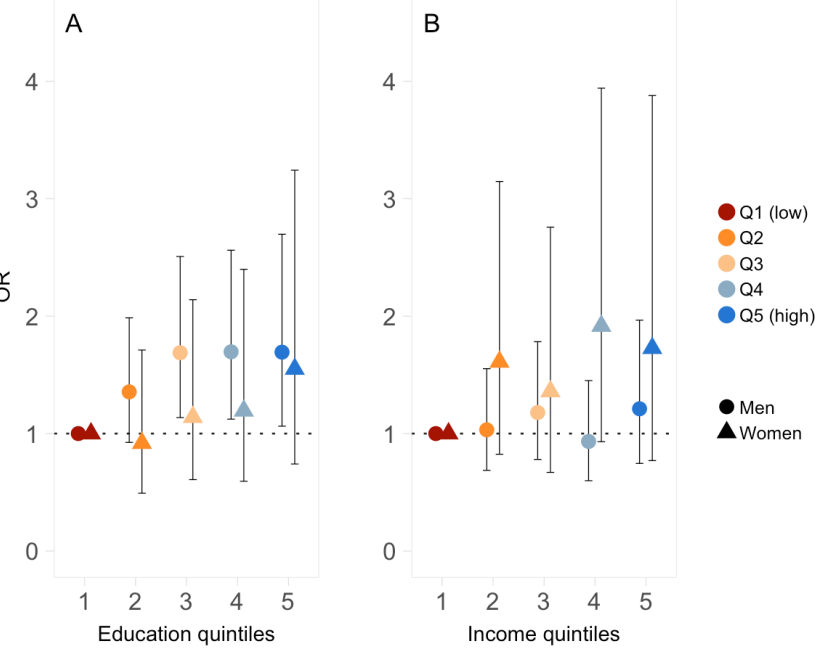

Figure 3 Relationship between area-level socioeconomic status and $O R$ for 30-day survival among men and women after multiple imputations. A, categorised by education quintiles; $B$, categorised by income quintiles.

\section{DISCUSSION}

The main finding in this study was that educational level was a strong predictor of 30-day survival after OHCA, whereas the effect of income appeared to be mediated by educational level. Our estimates show that the effect of educational level is-as judged by model coefficients-on par with that of bystander CPR and gender (ORs around 1.4 and 1.2, respectively). We did not find any significant interaction between the SES measures and gender, although the association between education and survival appeared to be stronger among men.

Our findings can shed light on previously contradictory findings concerning the relationship between SES and survival after OHCA, some of which have shown significant associations, ${ }^{8-12}$ whereas others have not. ${ }^{1314}$ Previous investigators have used different measures for SES in their studies, such as property tax values, ${ }^{9-11}$ deprivation indexes ${ }^{8} 12$ and median household income, ${ }^{13} 14$ and these studies were not adjusted for education. Our findings show that area-level education-which is strongly related to economic conditions-is at least in this population, a better predictor of OHCA survival. This result is in line with a previous finding of a significant effect of individual-level education after adjustment for occupation when studying a selected group of cases of OHCA with VF as initial rhythm. ${ }^{18}$

Although often used interchangeably, education, income and other measures of SES can tap into different mechanisms of survival after OHCA. As indicators of SES, income and wealth are the most direct measures of economic resources commanded by individuals and households. ${ }^{5}$ At the area level, they also characterise access to community-based resources and exposure to stressors. $^{19}$

Education, in addition to predicting income and wealth, has been argued to facilitate learnt effectiveness and control over 
one's life. ${ }^{20}$ In an OHCA situation, the victim could benefit from relatives/peers who may act more rationally when they witness suffering symptoms prior to cardiac arrest. They may be more likely to promptly call EMS and initiate earlier CPR, both of which are associated with an increased chance of survival. ${ }^{21}$ They may also communicate more effectively with the dispatcher, which may explain the shorter response times seen in this study. A recent study revealed that highly educated individuals were more likely to be currently trained in CPR, ${ }^{22}$ which is likely to result in higher quality resuscitation. Another study has however found a negative association between education and odds of receiving bystander $\mathrm{CPR},{ }^{23}$ which may indicate that there is regional variation in this relationship. Other factors that could explain our results are the higher chronic-disease burden in people with lower levels of education ${ }^{24}$ and a higher prevalence of smoking in such a group. ${ }^{25}$

We were not able to corroborate the results of a recent review that revealed an overall stronger relationship between SES and cardiovascular outcomes among women compared with men. ${ }^{7}$ In the present study, the association was positive but weaker (not significant) among women compared with men. It is therefore possible that the results reflect a lack of statistical power. In any case, the gender difference requires further investigation.

Lacking individual-level measures of SES, we used area-level measures of education and income. Because of misclassification of individual SES when using area-level SES ('ecological fallacy'), our results cannot be directly interpreted as being valid at an individual level, and individual-level measures are possibly stronger than the ones reported here. ${ }^{26}$ The ecological fallacy is likely to be more severe when the area of aggregation is large. We used smaller areas compared with those in previously published studies. There are more than 16000 grid cells in Stockholm County, which is significantly higher than the number of census tracts in any city.

The associations reported in this study can thus better approximate those at the individual level, in comparison with associations found in previous research. ${ }^{18}$ Besides serving as a proxy for individual education, living in a highly educated area may have an independent effect on survival after OHCA, ${ }^{526}$ which would go unnoticed if using individual-level measures only.

The differences in survival chances among people of high versus low SES call for action. Two treatments that benefit OHCA patients are early $\mathrm{CPR}^{21}$ and early defibrillation by an automated external defibrillator (AED). ${ }^{27}$ Efforts to aim both CPR training and public AED programmes towards low SES communities may help reduce the differences in the chance of survival after OHCA.

Further studies should concern the relationship between SES and survival after OHCA on an individual level, with sex-stratified analyses. Individual-level data should be accompanied by comorbidity data and data on in-hospital treatments.

\section{Strengths and limitations}

The current study has several strengths. We included structured data on all cases of EMS-treated OHCA over 10 years in Stockholm County. We excluded all cases where the person did not reside in Stockholm, which could otherwise bias the results in both directions. Although we used an area-level measure of SES, we assessed it using smaller predefined areas than in most other studies. To our knowledge, our study is the first to assess sex differences in the relationship between SES and survival after OHCA.
Key messages

What is already known on this subject?

- The relationship between socioeconomic status (SES) and survival after out-of-hospital cardiac arrest (OHCA) has not been extensively studied. Most studies have used a single measurement of SES.

\section{What might this study add?}

- This observational study using 10 years of OHCA data in Stockholm, Sweden, found significantly higher survival rates in areas with high proportion of university education(Q1 $=6.5 \%, \mathrm{Q} 5=9.6 \%)$ and areas with high median household income (Q1=6.3\%, Q5=10.5\%). After mutual adjustment, only area-level education was statistically significant, suggesting that area-level education may have a better predictive value compared with area-level income.

\section{How might this impact on clinical practice?}

- Information on SES measurement that predicts survival is important to understand the underlying causes of SES differences in OHCA. The results from this study can work as a basis for designing effective automated external defibrillator programmes as well as aim for cardiopulmonary resuscitation training to low-SES communities.

The study has several limitations. First, we did not have access to individual-level SES measures and therefore the possibility of ecological fallacies cannot be excluded. The socioeconomic variables/values were collected in 2013, and data were not available prior to that. It is possible that the socioeconomic characteristics of an area change during a 10 -year period, which we have not been able to take into account. Second, there is a possibility of residual confounding due to the study design. Third, we could not account for the total number of deaths in each area. Therefore there is a possibility of selection bias (eg, patients with high SES and low chance of survival were not registered).

\section{CONCLUSION}

In the present study we found a significant relationship between area-level education and 30-day survival after OHCA. Arealevel income did not independently predict 30-day survival after OHCA.

Correction notice Since this paper was first published online, figure 3 has been updated.

Contributors MJ, JHä, PN and PLSL designed the research. MJ and JHä performed statistical analysis. JHe, AR and MJ acquired the data. MJ, JH, PN, LS, JHe drafted the manuscript. All authors critically revised the manuscript. MJ is responsible for the overall content.

Funding The authors have not declared a specific grant for this research from any funding agency in the public, commercial or not-for-profit sectors.

Competing interests None declared.

Patient consent Not required.

Ethics approval The regional ethics committee in Stockholm (id 2017/716 - 31) .

Provenance and peer review Not commissioned; externally peer reviewed.

\section{REFERENCES}

1 Berdowski J, Berg RA, Tijssen JG, et al. Global incidences of out-of-hospital cardiac arrest and survival rates: Systematic review of 67 prospective studies. Resuscitation 2010;81:1479-87. 
2 Sasson C, Rogers MA, Dahl J, et al. Predictors of survival from out-of-hospital cardiac arrest: a systematic review and meta-analysis. Circ Cardiovasc Qual Outcomes 2010;3:63-81.

3 Kaplan GA, Keil JE. Socioeconomic factors and cardiovascular disease: a review of the literature. Circulation 1993;88:1973-98.

4 Clark AM, DesMeules M, Luo W, et al. Socioeconomic status and cardiovascular disease: risks and implications for care. Nat Rev Cardiol 2009;6:712-22.

5 Galobardes B, Shaw M, Lawlor DA, et al. Indicators of socioeconomic position (part 1). J Epidemiol Community Health 2006;60:7-12.

6 Geyer S, Hemström 0, Peter R, et al. Education, income, and occupational class cannot be used interchangeably in social epidemiology. Empirical evidence against a common practice. J Epidemiol Community Health 2006;60:804-10.

7 Backholer K, Peters SAE, Bots SH, et al. Sex differences in the relationship between socioeconomic status and cardiovascular disease: a systematic review and metaanalysis. J Epidemiol Community Health 2017;71:550-7.

8 Ahn KO, Shin SD, Hwang SS, et al. Association between deprivation status at community level and outcomes from out-of-hospital cardiac arrest: a nationwide observational study. Resuscitation 2011;82:270-6.

9 Clarke SO, Schellenbaum GD, Rea TD. Socioeconomic status and survival from out-ofhospital cardiac arrest. Acad Emerg Med 2005;12:941-7.

10 Vaillancourt C, Lui A, De Maio VJ, et al. Socioeconomic status influences bystander CPR and survival rates for out-of-hospital cardiac arrest victims. Resuscitation 2008;79:417-23.

11 Hallstrom A, Boutin P, Cobb L, et al. Socioeconomic status and prediction of ventricular fibrillation survival. Am J Public Health 1993;83:245-8.

12 Lee SY, Song KJ, Shin SD, et al. A disparity in outcomes of out-of-hospital cardiac arrest by community socioeconomic status: a ten-year observational study. Resuscitation 2018;126:130-6.

13 Galea S, Blaney S, Nandi A, et al. Explaining racial disparities in incidence of and survival from out-of-hospital cardiac arrest. Am J Epidemiol 2007;166:534-43.

14 Sayegh AJ, Swor R, Chu KH, et al. Does race or socioeconomic status predict adverse outcome after out of hospital cardiac arrest: a multi-center study. Resuscitation 1999;40:141-6.
15 Statistics Sweden, 2018. Statistikdatabasen. http://www.statistikdatabasen.scb.se/ (accessed 14 Mar 2018).

16 Jacobs I, Nadkarni V, Bahr J, et al. Cardiac arrest and cardiopulmonary resuscitation outcome reports: update and simplification of the Utstein templates for resuscitation registries: a statement for healthcare professionals from a task force of the International Liaison Committee on Resuscitation (American Heart Association, European Resuscitation Council, Australian Resuscitation Council, New Zealand Resuscitation Council, Heart and Stroke Foundation of Canada, InterAmerican Heart Foundation, Resuscitation Councils of Southern Africa). Circulation 2004; 110:3385-97.

17 Buuren S, Groothuis-Oudshoorn K. mice: multivariate imputation by chained equations in R. J Stat Softw 2011;45.

18 Wells DM, White LL, Fahrenbruch CE, et al. Socioeconomic status and survival from ventricular fibrillation out-of-hospital cardiac arrest. Ann Epidemiol 2016;26:418-23.

19 Diez Roux AV, Mujahid MS, Hirsch JA, et al. The Impact of Neighborhoods on CV Risk. Glob Heart 2016;11:353-63.

20 Mirowsky J, Education RCE. learned effectiveness and health. London Rev Educ 2005;3:205-20.

21 Hasselqvist-Ax I, Riva G, Herlitz J, et al. Early cardiopulmonary resuscitation in out-ofhospital cardiac arrest. N Engl J Med 2015;372:2307-15.

22 Blewer AL, Ibrahim SA, Leary M, et al. Cardiopulmonary resuscitation training disparities in the United States. J Am Heart Assoc 2017;6.

23 Straney LD, Bray JE, Beck B, et al. Are sociodemographic characteristics associated with spatial variation in the incidence of OHCA and bystander CPR rates? A population-based observational study in Victoria, Australia. BMJ Open 2016;6:e012434.

24 Braveman PA, Cubbin C, Egerter $S$, et al. Socioeconomic disparities in health in the United States: what the patterns tell us. Am J Public Health 2010;100:S186-96.

25 Huisman M, Kunst AE, Mackenbach JP. Inequalities in the prevalence of smoking in the European Union: comparing education and income. Prev Med 2005;40:756-64.

26 Galobardes B, Shaw M, Lawlor DA, et al. Indicators of socioeconomic position (part 2). J Epidemio/ Community Health 2006;60:95-101.

27 Valenzuela TD, Roe DJ, Nichol G, et al. Outcomes of rapid defibrillation by security officers after cardiac arrest in casinos. N Engl J Med 2000;343:1206-9. 